\title{
Evaluation of Oxidative Stress, Apoptosis, and Expression of MicroRNA-208a and MicroRNA-1 in Cardiovascular Patients
}

\author{
Asma Mohammadi ${ }^{1}$, Ali Reza Balizadeh Karami ${ }^{2}$, Vahid Dehghan Mashtani ${ }^{2}$, \\ Tooba Sahraei ${ }^{1}$, Zeinab Bandani Tarashoki ${ }^{2}$, Ehsan Khattavian² ${ }^{2}$ Sara Mobarak ${ }^{1}$, \\ Hossein Moradi Kazerouni ${ }^{2}$, Esmat Radmanesh ${ }^{* 1,2}$
}

\begin{abstract}
Background: MicroRNA expression signature and reactive oxygen species (ROS) production have been associated with the development of cardiovascular diseases (CVDs). This study aimed to evaluate oxidative stress, inflammation, apoptosis, and the expression of miRNA-208a and miRNA-1 in cardiovascular patients.

Methods: The study population included four types of patients (acute coronary syndromes (ACS), myocardial infarction (MI), arrhythmia, and heart failure (HF)), with 10 people in each group, as well as a control group. Quantitative real-time PCR was performed to measure mir-208 and miR-1 expression, the mRNAs of inflammatory mediators (TNFa, iNOS/eNOS), and apoptotic factors (Bax and Bcl2). XOX, MDA, and antioxidant enzymes (CAT, SOD, and GPx) were measured by ZellBio GmbH kits by an ELISA Reader.

Results: The results showed significant decreases in the activity of antioxidant enzymes (CAT, SOD, and Gpx) and a significant increase in the activity of the MDA and XOX in cardiovascular patients. Significant increases in IL-10, iNos, iNOS / eNOS, and TNF- $\alpha$ in cardiovascular patients were also observed. Also, a significant increase in the expression of miR-208 (HF> arrhythmia> ACS > MI) and a significant decrease in the expression of miR-1 (ACS > arrhythmia $>\mathrm{HF}>\mathrm{MI}$ ) were found in all four groups in cardiovascular patients.

Conclusions: The results showed increases in oxidative stress, inflammation, apoptotic factors, and in the expression of miR-208a in a variety of cardiovascular patients (ACS, MI, arrhythmia, and HF). It is suggested that future studies determine the relationships that miR-1, miR-208, and oxidative stress indices have with inflammation and apoptosis.
\end{abstract}

Keywords: Apoptosis, Cardiovascular diseases, Inflammation, microRNA-208a, microRNA-1, Oxidative stress.

\section{Introduction}

Cardiovascular diseases (CVDs) are a major cause of mortality and morbidity (1) and are currently the leading cause of death worldwide (2). CVDs encompass peripheral arterial disease, ischemic heart disease (IHD), stroke, heart failure (HF), and many other cardiac and vascular conditions (3).

Acute coronary syndrome (ACS) and coronary artery disease together account for approximately 7 million deaths each year (4). The prevalence of HF also increases with age (5). In adults who are middle-aged or older, conduction system diseases, arrhythmia, and atrial fibrillation account for most rhythm conditions (6).

CVDs are related to inflammation due to the close interaction between inflammation and oxidative stress (7). An increase in 
inflammatory cytokines is related to a higher risk of developing a CVD (8). Apoptosis has been detected in many CVDs, including myocardial ischemia, chronic HF, atherosclerosis, reperfusion injury, and diabetic cardiomyopathy (9). Bcl-2 family members can be anti-apoptotic (Bcl-XL, Bcl-2) or proapoptotic (Bid, Bax, Bak, and Bik). Plasma concentrations of $\mathrm{TNF} \alpha$ and catecholamines increase during HF. There is also evidence that toxic cardiomyopathies are related to cardiomyocyte apoptosis (10).

MicroRNAs (miRNAs) are small, noncoding RNAs. Distinct miRNA signatures have been reported in CVD and are potent modulators of diverse biological processes and pathologies (11). The most abundant miRNA in the heart is miR-1 (12). Thus, the upregulation and downregulation of miR-1 might result in abnormal heart function. This miRNA is encoded by two nearly identical genes (miR-1-1 and miR-1-2). Various heart abnormalities observed in mice involved a lack of the miR-12 gene and have been found to survive because they still produce some miR-1 from their remaining miR-1-1 gene. Mice have been produced that lack both miR-1 genes and die before weaning (13). The proper expression of miR-1 is necessary to maintain normal heart function. As such, increases or decreases in miR-1 expression can lead to many cardiac diseases (14). miR-208 is a highly conserved miRNA family with two members (miR-208a and miR-208b) (15). Gains and losses of the function of miR-208a are associated with arrhythmia, while the cardiac overexpression of miR-208a causes hypertrophy (16). Striking perturbations of plasma levels of heart-related miRNA-208b can cause diverse forms of myocardial damage in humans (17). Mirna expression signatures and reactive oxygen species (ROS) production have been linked to the development of CVDs $(18,19)$.

Oxidative stress plays an important role in several CVDs, such as hypertension, cardiac hypertrophy, atherosclerosis, and HF (20). The expression levels of many miRNAs can be altered by oxidative stress (21).
This study aimed to evaluate oxidative stress, inflammation, apoptosis, and the expression of miRNA-208a and miRNA-1 in four types of cardiovascular patients (acute coronary syndrome (ACS), myocardial infarction (MI), arrhythmia, and HF) and compare these patients with a control group.

\section{Materials and Methods}

This study was conducted in Abadan in the summer of 2017. A total of 137 cardiovascular patients $(38.3 \%$ ACS, $18.3 \%$ MI, $12.5 \%$ arrhythmia, and $12.5 \%$ HF) participated in the study. Their statuses were confirmed during the study period in Taleghani Hospital in Abadan by a cardiologist. Of the participants, $54.5 \%$ were male and $45.5 \%$ were female, with an average age of $64 \pm 13$ years. Furthermore, $35.2 \%$ of the patients had a history of smoking, $53.1 \%$ had a history of hypertension, and $42.3 \%$ had a history of diabetes. Ten participants from each group (50\% male and 50\% female) underwent blood sampling. The average age of the control group was $45 \pm 14$ years ( $50 \%$ male and $50 \%$ female); the members of this group had no history of any of the four studied disease types. The groups were matched by gender. All members of the patient groups and control group signed an informed consent form stating their agreement to participate in the study. The participants answered the questions from the researcher-made checklist, which contained questions about participants' demographic and clinical information. After patients completed the checklist, a blood sample was taken from them. Participants who did not want to donate their blood were excluded from the study. The blood samples of patients were used for molecular measurements and oxidative stress.

\section{$R N A$ extraction, $c D N A$ synthesis, and real time-PCR} RNA extraction was performed from plasma for miR-208 and miR-1 using a RNeasy Mini Kit (Roche, Germany) according to the manufacturer's protocol. Total RNA extracted from the plasma for other targets was assessed using a RiboEx (GeneAll Biotech, Seoul, South Korea) kit. The purity and concentration 
of RNA were measured using Nanodrop 2000 (Thermo Scientific). All RNA samples were stored at $-80{ }^{\circ} \mathrm{C}$ until further analysis. Firststrand cDNA was synthesized from total RNA using a hyper script TM first-strand synthesis kit (GeneAll, Korea). Gene expression assessments were made using a Lightcycler96 real-time PCR system (Roche, Germany) made by SYBR Premix Ex Taq TM (Takara, Japan). The relative quantitative real-time PCR method was employed to Bax, Bcl-2, iNOS, eNOS, IL-10, TNF- $\alpha$, miR-1, and miR-208 gene expression levels. GAPDH and U6 were used as an endogenous control to normalize the quantities of the target genes. Data were analyzed using the comparison $\mathrm{Ct}(2-\Delta \Delta \mathrm{Ct})$ method and expressed as fold changes in comparison with respective control data (22, 23). The sequences of primers are listed in Table 1.

Table 1. The sequences of primers

\begin{tabular}{ll}
\hline \multirow{2}{*}{ Gene } & Primer Sequence (5'-3') \\
\hline \multirow{2}{*}{ Bax } & $\begin{array}{l}\text { Forward: 5'-GCTTCAGGGTTTCATCCAG -3' } \\
\text { Reverse: 5'-GGCGGCAATCATCCTCTG- 3' }\end{array}$ \\
\hline \multirow{2}{*}{ Bcl-2 } & $\begin{array}{l}\text { Forward: 5'-TACTTAAAAAATACAACATCACAG- 3' } \\
\text { Reverse: 5'-GGAACACTTGATTCTGGTG -3' }\end{array}$ \\
\hline \multirow{2}{*}{ TNF- $\alpha$} & $\begin{array}{l}\text { Forward: 5'-CCCAGGGACCTCTCTCTAATC -3' } \\
\text { Reverse: 5'-ATGGGCTACAGGCTTGTCACT- 3' }\end{array}$ \\
\hline \multirow{2}{*}{ IL-10 } & Forward: 5'-CATCGATTTCTTCCCTGTGAA -3' \\
\hline \multirow{2}{*}{ eNOS } & Reverse: 5'-TCTTGGAGCTTATTAAAGGCATTC -3' \\
\hline \multirow{2}{*}{ iNOS } & $\begin{array}{l}\text { Forward: 5'-CAGTGTCCAACATGCTGCTGGAAATTG-3' } \\
\text { Reverse: 5'-TAAAGGTCTTCTTCCTGGTGATGCC-3' }\end{array}$ \\
\hline
\end{tabular}

\section{Biochemical tests}

Blood samples were collected and centrifuged at $4000 \mathrm{~g}$ for $10 \mathrm{~min}$; the plasma was kept at -80 ${ }^{\circ} \mathrm{C}$ for biochemical analysis, MDA, XOX, and antioxidant enzymes (CAT, SOD, and GPx) were measured by Zell bioGmbH kits (Germany) by ELISA Reader (BioTek, U.S.A).

\section{Statistical analysis}

The data were analyzed using GraphPad Prism 8 and were expressed as mean \pm SEM. One-way analysis of variance (ANOVA) followed by LSD was used for comparison tests, and $\mathrm{p}<0.05$ was considered statistically significant.

\section{Results}

MDA levels in the ACS, HF, MI, and arrhythmia groups increased significantly in comparison to the control group, and the highest MDA levels were observed in the MI group. After that, the highest levels of MDA were observed in the arrhythmia and $\mathrm{HF}$ groups, and the lowest levels were observed in the ACS group (Fig. 1A).

The enzyme activity of xanthine oxidase in the ACS, HF, MI, and arrhythmia groups exhibited a significant increase when compared to the control group. The highest amount of XOX was observed in the arrhythmic group, followed by the MI group. The lowest amount of XO was observed in the ACS group (Fig. 1B).

A significant decrease in catalase activity was observed in all four patient groups, with the lowest activity of this enzyme observed in the arrhythmia group (Fig. 2A). A significant decrease in the amount of the GPX enzyme was observed in all patient groups when compared to the control group. The lowest amount of this enzyme was observed in HF groups, followed by the arrhythmia group; the highest amount was observed in the ACS 
group (Fig. 2B). The SOD enzyme activity in all patient groups showed a significant decrease when compared to the control group. The lowest amount of this enzyme was observed in the HF group (Fig. 2C).

A:

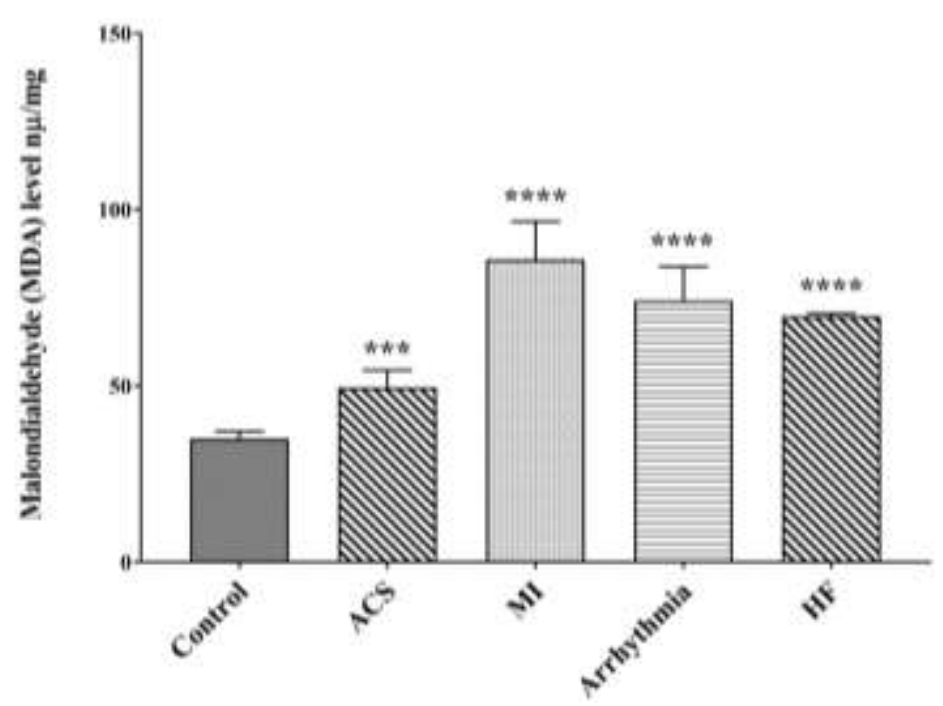

B:

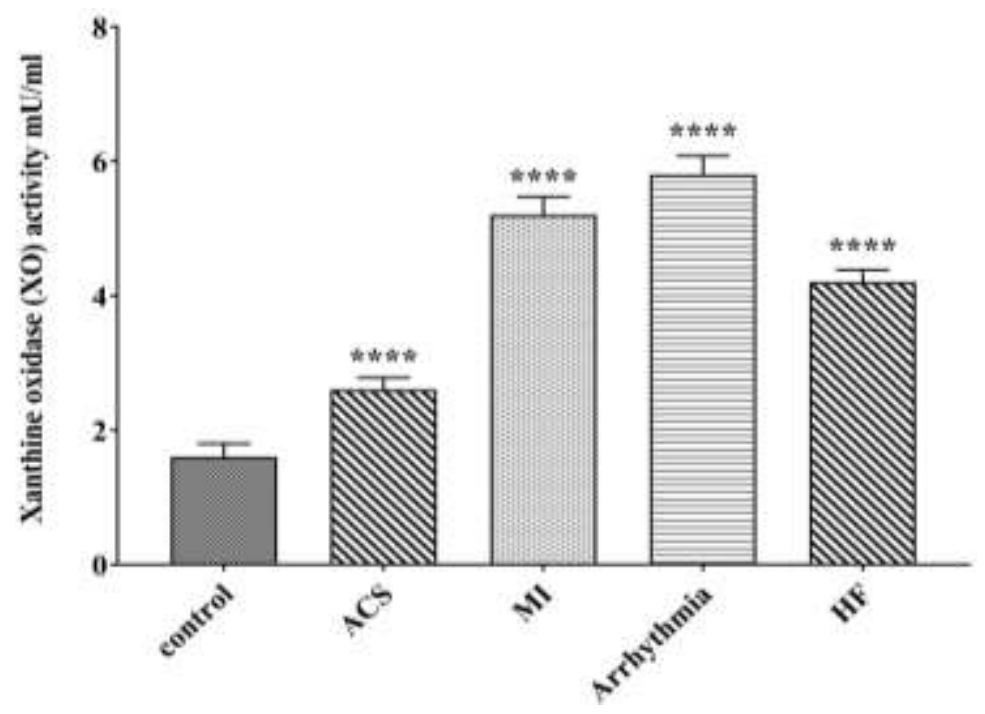

Fig. 1. Comparison of A: MDA and B: XOX level in control, ACS, MI, Arrhythmia, and HF groups. For the total test one-way ANOVA followed by Fisher s LSD test was used; ****p< 0.0001 Vs. control group. 
A:

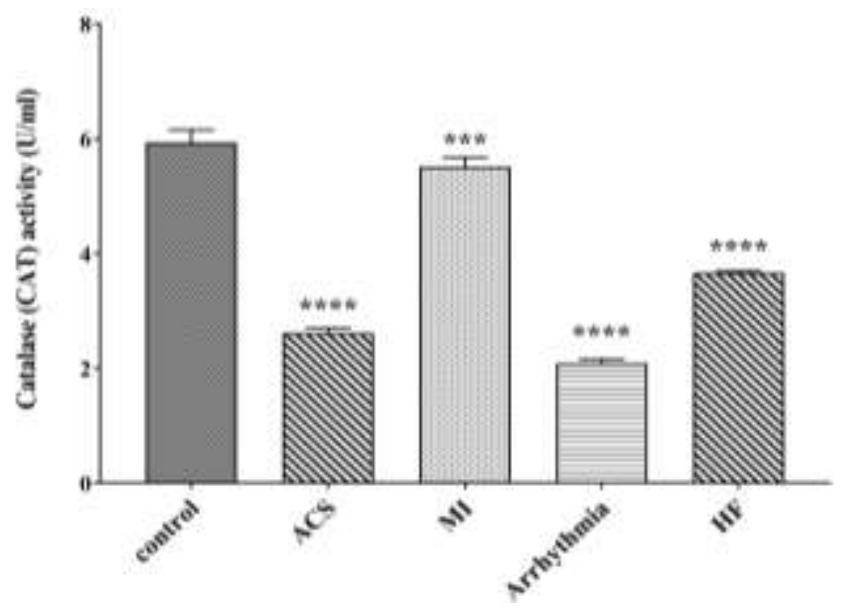

B:

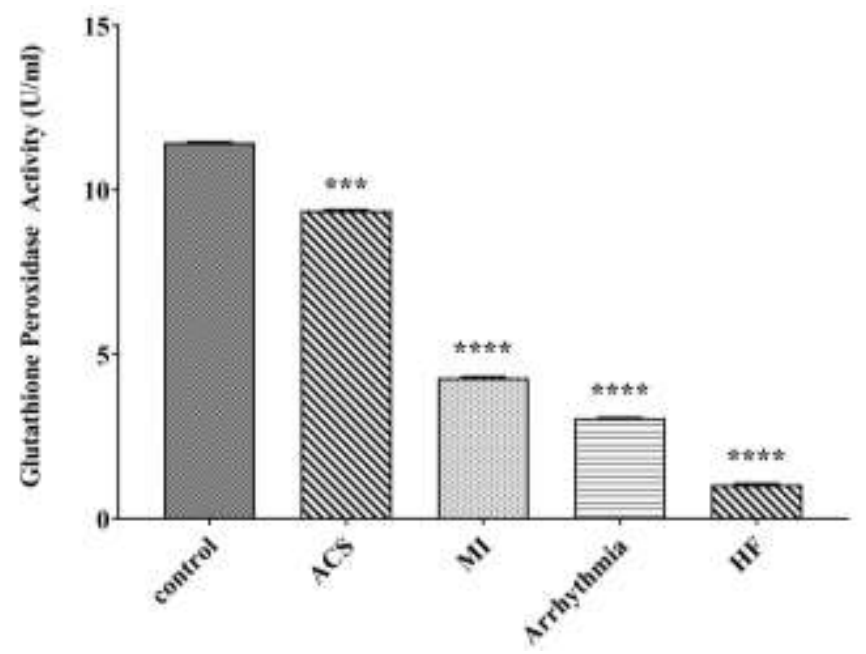

C:

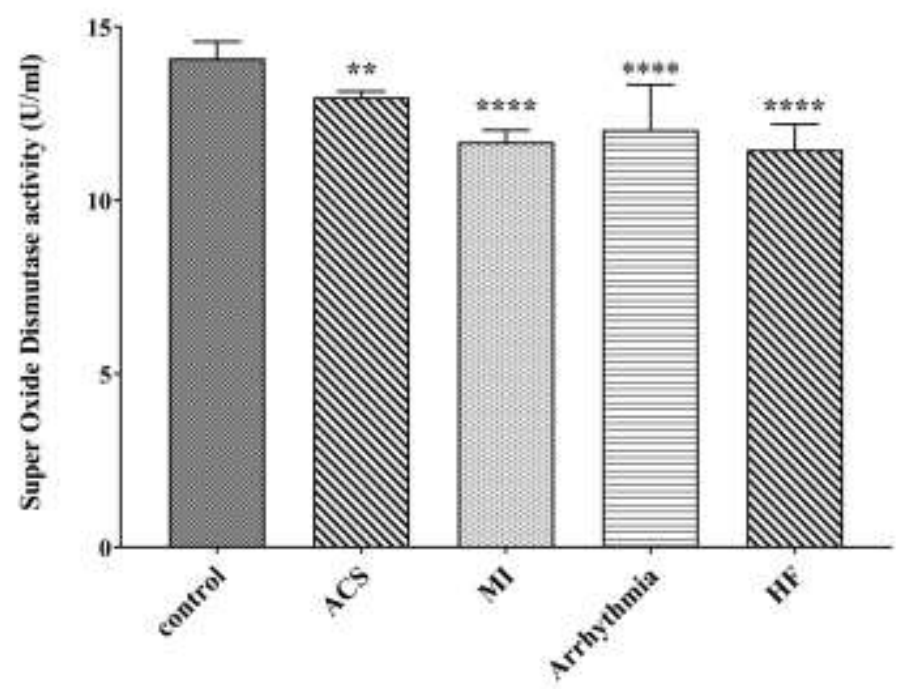

Fig. 2. Comparison of A: activity CAT enzyme, B: activity GPX enzyme and C: activity SOD enzyme in control, ACS, MI, Arrhythmia, and HF groups. For the total test one way ANOVA followed by Fisher s LSD test was used; $* * \mathrm{p}<0.01$, ****p< 0.0001 Vs. control group. 
The expression of miR-1 in all patient groups showed a significant decrease when compared to the control group. Among the patient groups, the lowest rate was observed in the MI group, while the highest rate was observed in the ACS group (Fig. 3A). A significant increase in miR-208 expression was observed in all patient groups when compared to the control group, with the lowest rate observed in the MI group and the highest observed in the HF group (Fig. 3B).

The expression of the eNOS gene in all four groups of heart patients showed a significant decrease compared to that of the control group (Fig. 4A). The level of iNOS expression in the groups with ACS, HF, and MI showed a significant increase compared to that of the control group, and the highest level of iNOS A:

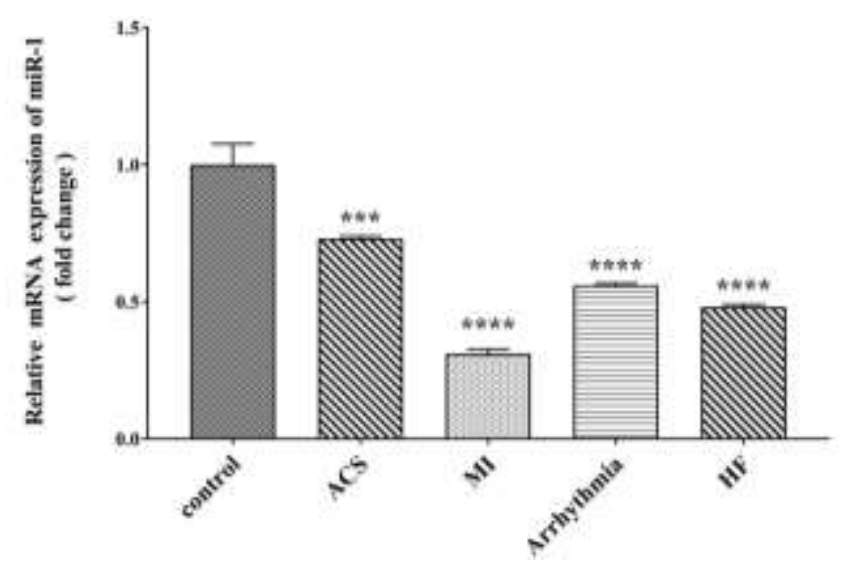

B:

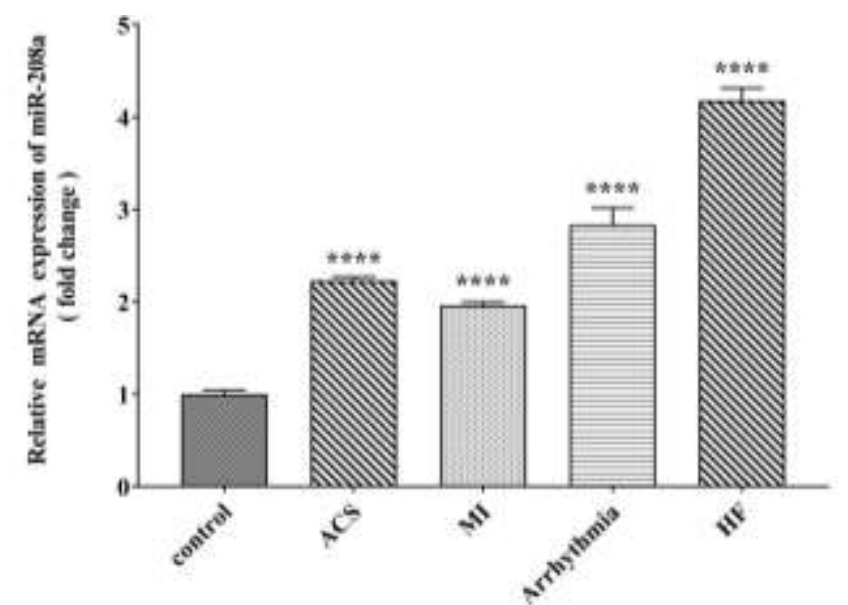

Fig. 3. Comparison of A: relative mRNA expression of miR- 1 (fold change) and B: relative mRNA expression of miR- 208a (fold change) in control, ACS, MI, Arrhythmia and HF groups. For the total test one way ANOVA followed by Fisher s LSD test was used; $* * * p<0.001$, ****p $<0.0001$ Vs. control group. expression was observed in the group with ACS (Fig. 4B). The ratio of iNOS / eNOS expression in all four groups of heart patients compared to that of the control group showed a significant increase, with the highest increase in the group of heart failure and the lowest ratio in the group with arrhythmia (Fig. 4C).

Interleukin 10 gene expression was significantly increased in each group of heart patients compared to that of the control group and the highest rate was observed in the groups with heart failure and ACS, and the lowest rate was identified in the arrhythmia group (Fig. $5 \mathrm{~A}$ ). The expression level of the TNF- $\alpha$ gene in all four groups with heart disease showed a significant increase compared to that of the control group, and the highest rate was detected in the group with ACS (Fig. 5B). 
A:

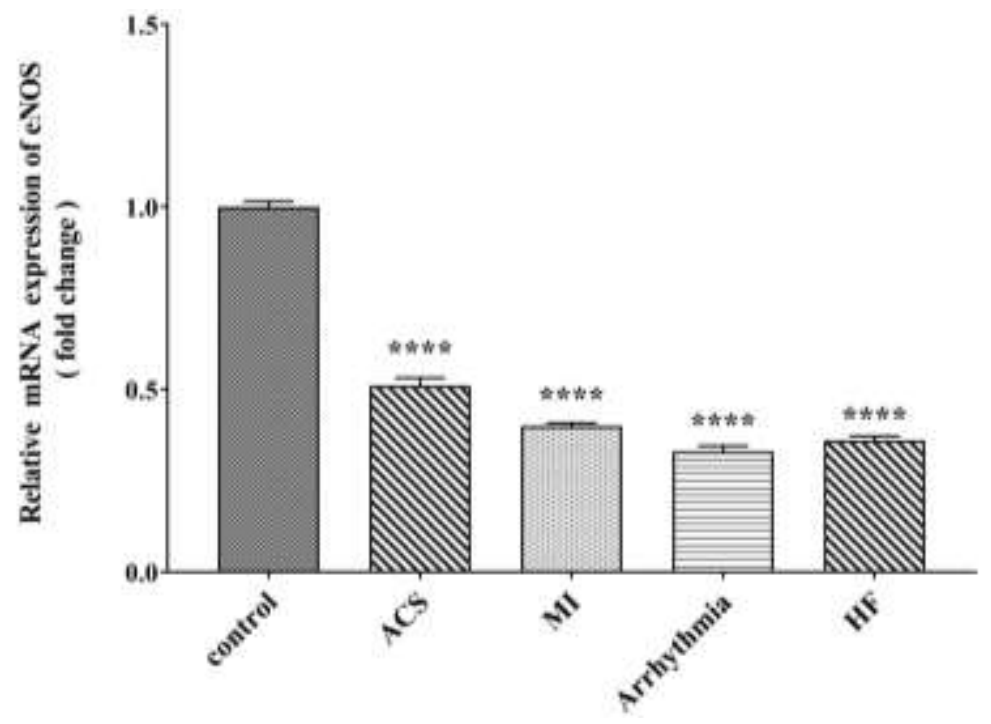

B:

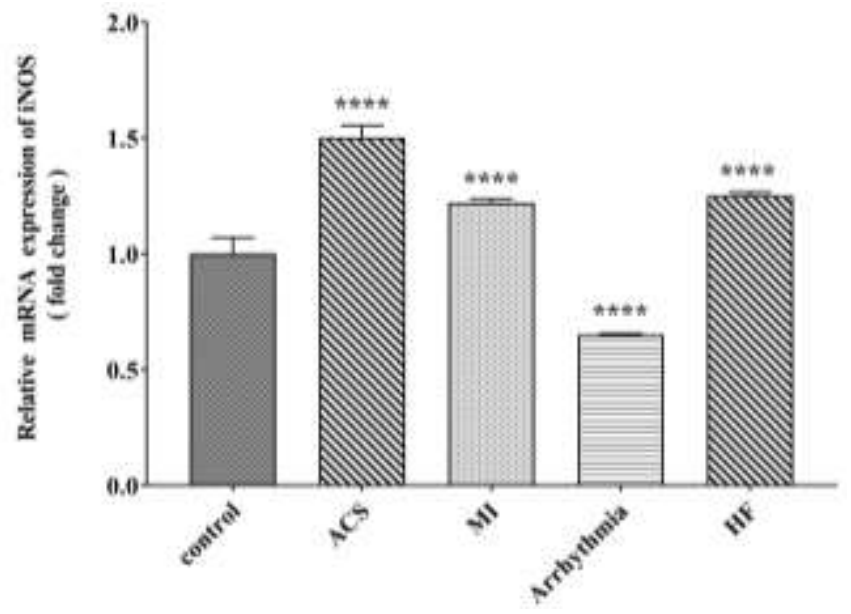

C:

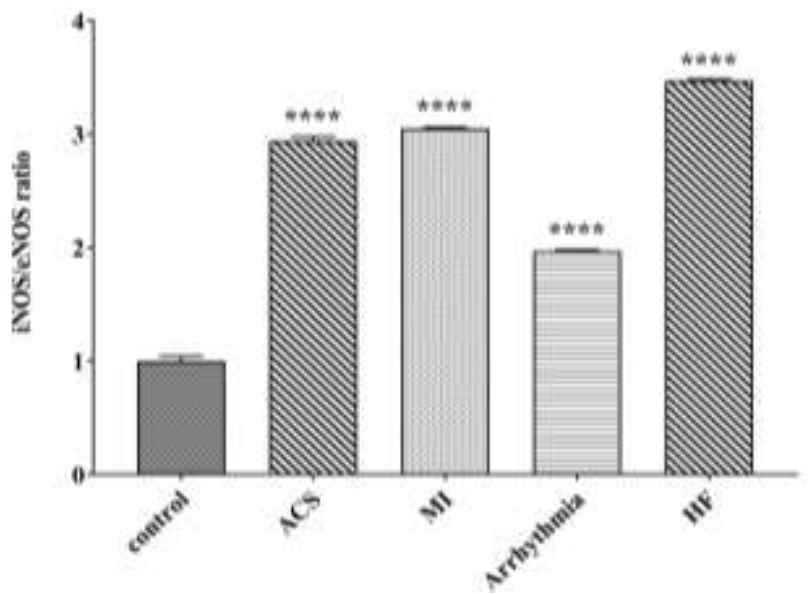

Fig. 4. Comparison of A: relative mRNA expression of eNOS (fold change) B: relative mRNA expression iNOS (fold change) and C: iNOS/eNOS ratio in control, ACS, MI, Arrhythmia and HF groups. For the total test one way ANOVA followed by Fisher s LSD test was used; $* * * * p<0.0001$ Vs. control group. 
A:

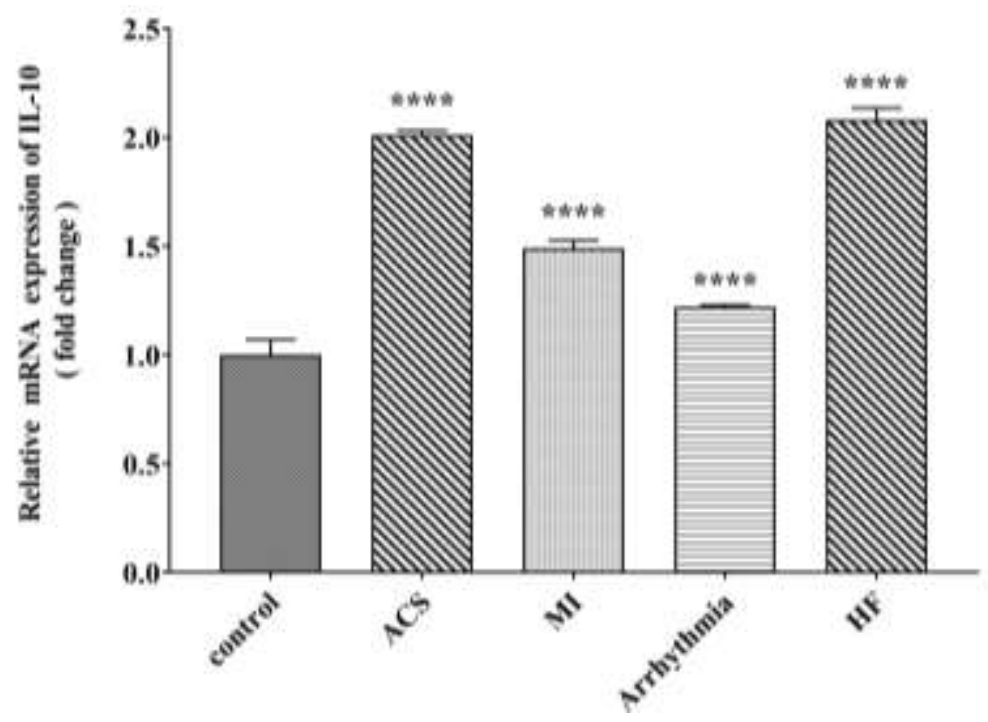

B:

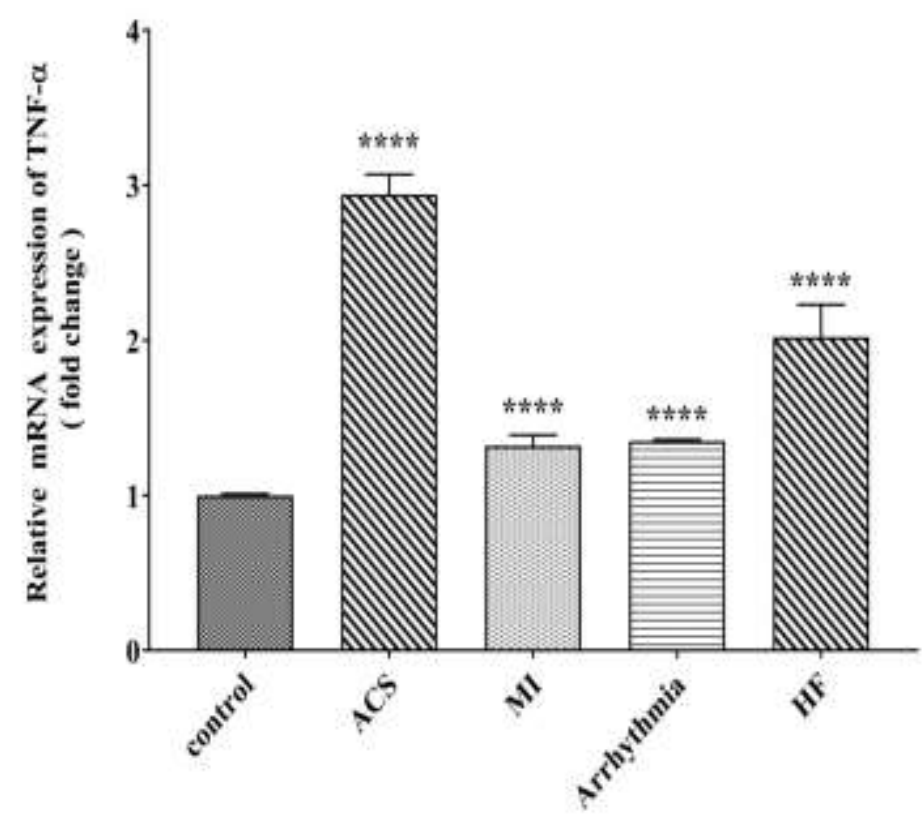

Fig. 5. Comparison of A: relative mRNA expression of IL-10 (fold change), B: relative mRNA expression of TNF- $\alpha$ (fold change) in control, ACS, MI, Arrhythmia, and HF groups. For the total test one way ANOVA followed by Fisher s LSD test was used; ****p $<0.0001$ Vs. control group.

The expression of the BAX gene in the four groups of heart patients showed a significant increase compared to that of the control group, and the highest rate was observed in the group with ACS, and the lowest rate was observed in the group with arrhythmia (Fig. 6A). A significant decrease in Bcl-2 gene expression was observed in all four groups of heart patients compared to that of the control group (Fig. 6B). A significant increase in $\mathrm{BAX} / \mathrm{Bcl}-2$ ratio was observed in all four groups of heart patients, which was the highest in the MI group (Fig. $6 \mathrm{C})$. 
A:

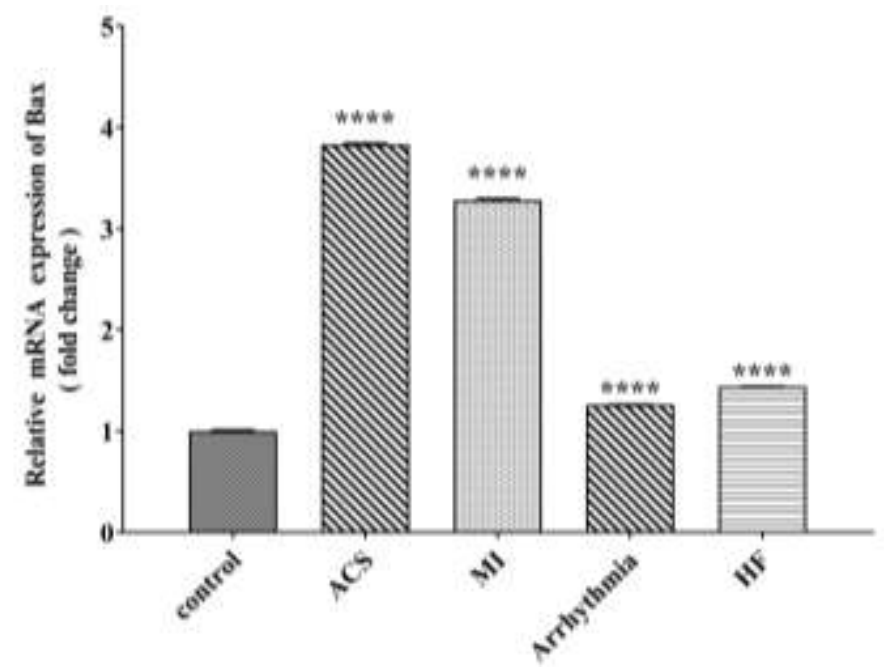

B:

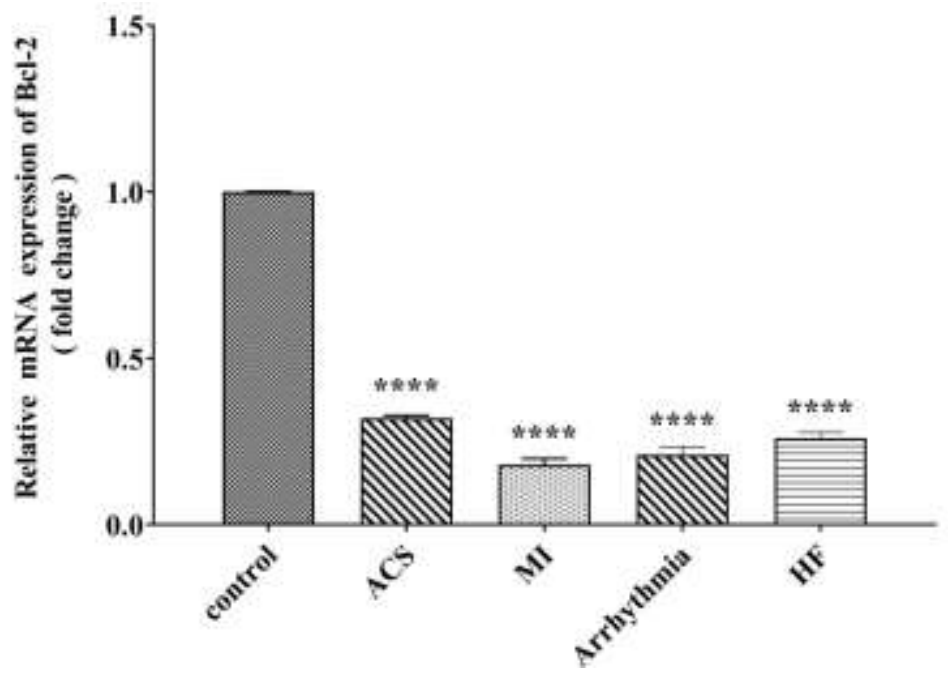

C:

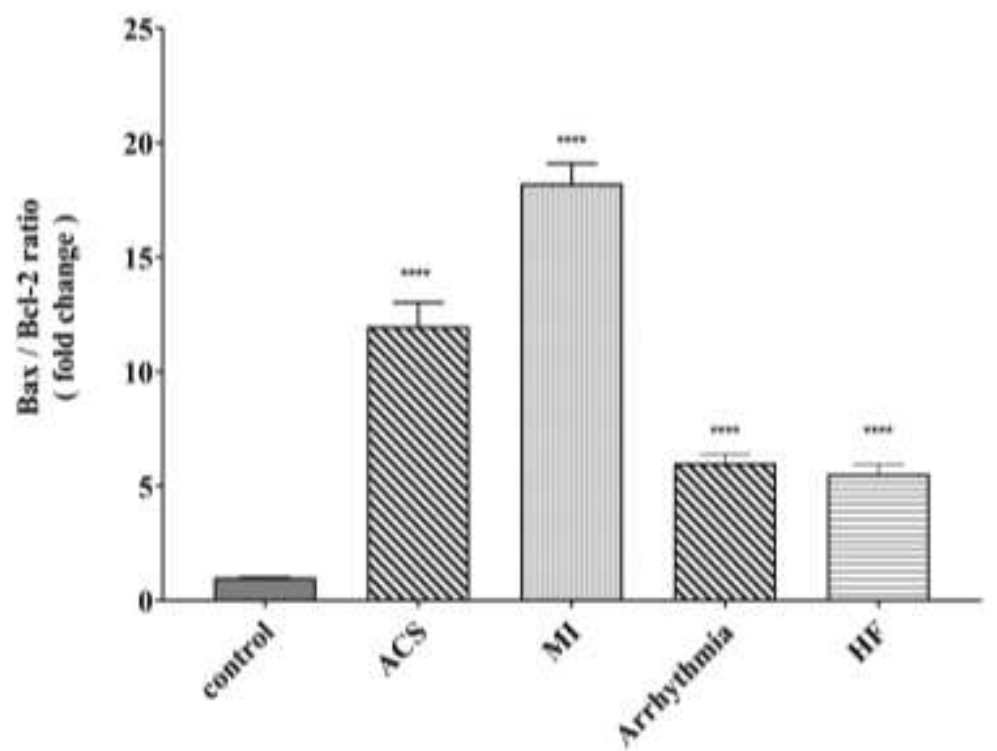

Fig. 6. Comparison of A: relative mRNA expression of Bax (fold change), B: relative mRNA expression of Bcl-2 (fold change), and C: of Bax/Bcl-2 ratio in control, ACS, MI, Arrhythmia and HF groups. For the total test one-way ANOVA followed by Fisher s LSD test was used; ****p< 0.0001 Vs. control group. 


\section{Discussion}

In this study, significant decreases in the activity of antioxidant enzymes (CAT, SOD, and Gpx) were observed in cardiovascular patients. Also, a significant decrease in eNOS expression was observed in cardiovascular patients when compared to a control group. Significant increases in inflammatory factors IL-10 (HF> ACS> MI> arrhythmia), iNos, iNOS / eNOS (HF> ACS> MI> arrhythmia), and TNF- $\alpha$ (ACS $>\mathrm{HF}>$ arrhythmia $>\mathrm{MI})$ in cardiovascular patients were also observed in this study.

The rate of frequent cardiovascular events was lowered by targeting interleukin $1 \beta$ using a specific monoclonal antibody (Canakinumab) in patients with previous myocardial infarction (8).

Cardiac reserve post-MI is improved by an iNOS deficiency, caused by a reduction in oxidative stress (24). iNOS expression was found to associate with the severity of cardiac dysfunction (25).

There was a significant increase in apoptosis factors, such as BAX and BAX / Bcl-2 ratio, in cardiovascular patients (MI> ACS> arrhythmia> HF) when compared to the control group.

The overexpression of TNF- $\alpha$ in the heart results in increased apoptosis and HF risk. Progressive LV wall thinning and dilation was accompanied by a rise in cardiomyocyte apoptosis, and a progressive loss of Bcl-2 was seen in transgenic mice with overexpression TNF in the heart (26).

There was a significant increase in the expression of miR-208a in all four groups in cardiovascular patients when compared to the control group in the following order: HF> arrhythmia > ACS > MI).

In another study, it was seen that in the hearts of MI patients, when compared to healthy adult and fetal hearts, miR-1 and miR133a were downregulated, whereas miR-208 was upregulated (27). In response to several forms of cardiac stress, miR-208-null mice did not show fibrosis or cardiomyocyte hypertrophy (28). The levels of miR-208b and miR-499-5p were significantly higher in both non-ST elevated myocardial infarction (NSTEMI) and elevated myocardial infarction (STEMI) patients when compared to non-MI patients. The levels of miR-1 were higher in NSTEMI patients than those in the non-MI group (29).

The levels of miR-208, miR-1, miR-134, miR-186, miR-223, and miR-499 in acute myocardial infarction (AMI) samples increased, and in 182 angina pectoris (AP) patients, mir-1 and mir-208 levels were higher when compared to the control group (30).

These results suggest that there was a significant decrease in the expression of miR-1 in cardiovascular patients than in the control group; the lowest rate was observed in the MI group (ACS > arrhythmia > HF> MI).

MiR-1 expression rates were significantly lower in elderly patients with atrial fibrillation (31). Further, miR-1 was downregulated in patients with symptomatic HF (32). The results of our study also showed a significant decrease in the expression of miR-1 in arrhythmia patients.

In an animal study based on the model of induction of acute myocardial in rat, was increased plasma level of miR-1 (33). In 82 patients with unstable angina (UA), mir-1 was upregulated when compared to the control group, whereas downregulation was observed in 130 cases (34).

Intracellular ROS can either induce or inhibit miRNA expression level (35). There was also a significant increase in the activity of the MDA group (MI> arrhythmia $>\mathrm{HF}>\mathrm{ACS}$ ) and XOX group (arrhythmia $>$ MI $>\mathrm{HF}>\mathrm{ACS}$ ) in cardiovascular patients when compared to the control group. In another study, a significant decrease in plasma MDA was observed following primary percutaneous transluminal coronary angioplasty (PTCA) (36). The specific activity of xanthine oxidase and MDA increases in patients with MI when compared to the control group (37). In congestive HF (CHF) patients, according to the New York Heart Association (NYHA), plasma levels of MDA are significantly higher than in healthy people. Class II NYHA patients showed significantly 
higher levels of vitamin $\mathrm{E}$, vitamin $\mathrm{A}$, lutein, and lycopene and significantly lower MDA levels than class III patients (38).

SOD had decreased in the AMI uncomplicated with arrhythmia group, and MDA had increased in the AMI complicated with arrhythmia group (39). The results of this study showed a significant decrease in the activity of antioxidant enzymes (CAT (MI> $\mathrm{HF}>\mathrm{ACS}>$ arrhythmia), SOD (ACS> arrhythmia $>\mathrm{MI}>\mathrm{HF}$ ) and Gpx (ACS $>\mathrm{MI}>$ arrhythmia $>$ HF) was in cardiovascular patients when compared to the control group.

Decreases the level of antioxidants, increases the risk of cardiovascular events in patients with atrial fibrillation (40). In another study, paroxysmal atrial fibrillation was characterized by significantly increased activity in SOD and CAT, therefore changes in oxidative status are closely correlated to CVD (39). Increased oxidative stress in human end-stage HF may lead to specific rearrangement of catalase gene expression as a compensatory mechanism (41). In a previous study, no differences were seen in terms of superoxide dismutase activity, although glutathione peroxidase activity was lower in the atrial fibrillation (AF) group (42). Elevated miR-1 levels have been observed in coronary artery disease and rats with myocardial infarction, both of which are associated with oxidative stress (20).

miRNAs can be considered potential targets and modulators of oxidative stress (43). Several miRNAs have been identified as oxidative stress-responsive miRNAs through the study of miRNA expression signatures that are associated with oxidative stress-related pathways (44). The crosstalk between ROS, CVDs, and miRNAs, with the potential to ameliorate or inhibit the progression of CVDs, could lead to new therapies based on

\section{References}

1. Hinton W, McGovern A, Coyle R, Han TS, Sharma $\mathrm{P}$, Correa $\mathrm{A}$, et al. Incidence and prevalence of cardiovascular disease in English primary care: a cross-sectional and follow-up study of the Royal College of General suppressing the effects of ROS (21). The results of this study revealed an increase in oxidative stress, inflammation, and apoptotic factors in four types of cardiovascular patients (acute coronary syndromes (ACS), myocardial infarction (MI), arrhythmia, and HF). A significant increase in the expression of miR208 was also observed in these patients, as was a significant decrease in the expression of miR-1.

It is suggested that future studies determine the relationships of miR-1, miR208a, and oxidative stress indices with inflammation and apoptosis. Such studies will certainly be helpful for the treatment of these patients.

In most previous studies, the expression of miR-208 in cardiovascular patients has been higher than in the control group. The results of our study were consistent with such findings. However, in the case of miR-1, different results have been reported. In some studies, miR-1 levels were higher in cardiovascular patients than in the control group, while in other studies, miR-1 levels were lower in patients than in the control group (the latter was found in our study as well). Further studies on miR expression in cardiovascular patients should be performed to resolve this inconsistency.

\section{Acknowledgements}

This study was a funded research project (96U-12) supported by Abadan Faculty of Medical Sciences, Abadan, Iran, with an ethical approval:

IR.ABADANUMS.REC.1396.219.

The authors gratefully acknowledge the Taleghani and Beheshti educational hospitals staff in Abadan city.

The authors declare no conflict of interest.

Practitioners (RCGP) Research and Surveillance Centre (RSC). BMJ Open. 2018;8(8):e020282.

2. Senoner T, Dichtl W. Oxidative Stress in Cardiovascular Diseases: Still a Therapeutic Target?. Nutrients. 2019;11(9):2090. 
3. Mensah GA, Roth GA, Fuster V. The Global Burden of Cardiovascular Diseases and Risk Factors: 2020 and Beyond. J Am Coll Cardiol. 2019;74(20):2529-2532.

4. Chan MY, Du X, Eccleston D, Ma C, Mohanan PP, Ogita M, et al. Acute coronary syndrome in the Asia-Pacific region. Int $\mathbf{J}$ Cardiol. 2016;202:861-9.

5. Benjamin EJ, Muntner $\mathrm{P}$, Alonso A, Bittencourt MS, Callaway CW, Carson AP, et al. Heart Disease and Stroke Statistics-2019 Update: A Report From the American Heart Association. Circulation. 2019;139(10):e56-e528.

6. Khurshid S, Choi SH, Weng LC, Wang EY, Trinquart L, Benjamin EJ, et al. Frequency of Cardiac Rhythm Abnormalities in a Half Million Adults. Circ Arrhythm Electrophysiol. 2018;11(7):e006273.

7. Steven S, Frenis K, Oelze M, Kalinovic S, Kuntic M, Bayo Jimenez MT, et al. Vascular Inflammation and Oxidative Stress: Major Triggers for Cardiovascular Disease. Oxid Med Cell Longev. 2019;2019:7092151.

8. Ridker PM, Everett BM, Thuren T, MacFadyen JG, Chang WH, Ballantyne C, et al. Antiinflammatory Therapy with Canakinumab for Atherosclerotic Disease. N Engl J Med. 2017;377(12):1119-1131.

9. Lee Y, Gustafsson AB. Role of apoptosis in cardiovascular disease. Apoptosis. 2009;14(4):536-48.

10. Bennett MR. Apoptosis in the cardiovascular system. Heart. 2002;87(5):480-487.

11. Zampetaki A, Mayr M. MicroRNAs in Vascular and Metabolic Disease. Circ Res. 2012;110(3):508-22.

12. Heidersbach A, Saxby C, Carver-Moore K, Huang Y, Ang YS, de Jong PJ, et al. microRNA1 regulates sarcomere formation and suppresses smooth muscle gene expression in the mammalian heart. Elife. 2013;2:e01323.

13. Ai J, Zhang R, Gao X, Niu HF, Wang N, Xu $\mathrm{Y}$, et al. Overexpression of microRNA-1 impairs cardiac contractile function by damaging sarcomere assembly. Cardiovasc Res. 2012;95(3):385-93.

14. Li J, Dong X, Wang Z, Wu J. MicroRNA-1 in Cardiac Diseases and Cancers. Korean $\mathrm{J}$ Physiol Pharmacol. 2014;18(5):359-363.
15. Vagner Oliveira-Carvalho VOC, and Edimar Alcides Bocchi. The Emerging Role of miR208a in the Heart. DNA Cell Biol. 2013;32(1):812.

16. Callis T, Pandya K, Seok H, Tang R-H, Tatsuguchi M, Huang Z-P, et al. MicroRNA208a is a regulator of cardiac hypertrophy and conduction in mice. $\mathbf{J}$ Clin Invest. 2009;119(9):2772-86.

17. Corsten MF, Dennert R, Jochems S, Kuznetsova T, Devaux Y, Hofstra L, et al. Circulating MicroRNA-208b and MicroRNA499 reflect myocardial damage in cardiovascular disease. Circ Cardiovasc Genet. 2010;3(6):499506.

18. Li M, Duan L, Li Y, Liu B. Long noncoding RNA/circular noncoding RNA-miRNA-mRNA axes in cardiovascular diseases. Life Sci. 2019;233:116440.

19. Hansen TB, Jensen TI, Clausen BH, Bramsen JB, Finsen B, Damgaard CK, et al. Natural RNA circles function as efficient microRNA sponges. Nature. 2013;495:384-388.

20. Wang L, Yuan Y, Li J, Ren H, Cai Q, Chen $\mathrm{X}$, et al. MicroRNA-1 aggravates cardiac oxidative stress by post-transcriptional modification of the antioxidant network. Cell Stress Chaperones. 2015;20(3):411-20.

21. Kura B, Szeiffova Bacova B, Kalocayova B, Sykora M, Slezak J. Oxidative StressResponsive MicroRNAs in Heart Injury. Int $\mathbf{J}$ Mol Sci. 2020;21(1):358.

22. Bayatiani MR, Ahmadi A, Aghabozorgi R, Seif F. Concomitant Up-Regulation of Hsa- Mir374 and Down-Regulation of Its Targets, Gsk-3 $\beta$ and Apc, in Tissue Samples of Colorectal Cancer. Rep Biochem Mol Biol. 2021;9(4):408416.

23. Shaker O, Mahfouz H, Salama A, Medhat E. Long Non-Coding HULC and miRNA-372 as Diagnostic Biomarkers in Hepatocellular Carcinoma. Rep Biochem Mol Biol. 2020;9(2):230-240.

24. Liu YH, Carretero OA, Cingolani OH, Liao $\mathrm{TD}$, Sun Y, Xu J, et al. Role of inducible nitric oxide synthase in cardiac function and remodeling in mice with heart failure due to myocardial infarction. Am J Physiol Heart Circ Physiol. 2005;289(6):H2616-23. 
25. Ferdinandy P, Danial H, Ambrus I, Rothery RA, Schulz R. Peroxynitrite is a major contributor to cytokine-induced myocardial contractile failure. Circ Res. 2000;87(3):241-7.

26. Haudek SB, Taffet GE, Schneider MD, Mann DL. TNF provokes cardiomyocyte apoptosis and cardiac remodeling through activation of multiple cell death pathways. J Clin Invest. 2007;117(9):2692-701.

27. Bostjancic E, Zidar N, Stajer D, Glavac D. MicroRNAs miR-1, miR-133a, miR-133b and miR-208 are dysregulated in human myocardial infarction. Cardiology. 2010;115(3):163-9.

28. Montgomery RL, Hullinger TG, Semus HM, Dickinson BA, Seto AG, Lynch JM, et al. Therapeutic inhibition of miR-208a improves cardiac function and survival during heart failure. Circulation. 2011;124(14):1537-47.

29. Gidlöf O, Smith J, Miyazu K, Gilje P, Spencer A, Blomquist $\mathrm{S}$, et al. Circulating cardio-enriched microRNAs are associated with long-term prognosis following myocardial infarction. BMC Cardiovasc Disord. 2013;13:12. 30. Li C, Fang Z, Jiang T, Zhang Q, Liu C, Zhang $C$, et al. Serum microRNAs profile from genome-wide serves as a fingerprint for diagnosis of acute myocardial infarction and angina pectoris. BMC Med Genomics. 2013;6:16.

31. Li YD, Hong YF, Yusufuaji Y, Tang BP, Zhou XH, Xu GJ, et al. Altered expression of hyperpolarization-activated cyclic nucleotidegated channels and microRNA-1 and -133 in patients with age-associated atrial fibrillation. Mol Med Rep. 2015; 12(3) 3243-3248.

32. Sygitowicz G, Tomaniak M, Błaszczyk O, Kołtowski Ł, Filipiak KJ, Sitkiewicz D. Circulating microribonucleic acids miR-1, miR21 and miR-208a in patients with symptomatic heart failure: Preliminary results. Arch Cardiovasc Dis. 2015;108(12):634-42.

33. Sun T, Zhang L, Li X, Chen F, Li Y, Ma X, et al. MicroRNA-1 and Circulating Microvesicles Mediate the Protective Effects of Dantonic in Acute Myocardial Infarction Rat Models. Front Physiol. 2018;9:664.

34. Li S, Sun YN, Zhou YT, Zhang CL, Lu F, Liu J, et al. Screening and identification of microRNA involved in unstable angina using gene-chip analysis. Exp Ther Med. 2016;12(4):2716-2722.

35. Banerjee J, Khanna S, Bhattacharya A. MicroRNA Regulation of Oxidative Stress. Oxid Med Cell Longev. 2017;2017:2872156.

36. Olsson KA, Harnek J, Ohlin AK, Pavlidis N, Thorvinger B, Ohlin $\mathrm{H}$. No increase of plasma malondialdehyde after primary coronary angioplasty for acute myocardial infarction. Scand Cardiovasc J. 2002;36(4):237-40.

37. Raghuvanshi R, Kaul A, Bhakuni P, Mishra A, Misra MK. Xanthine oxidase as a marker of myocardial infarction. Indian $\mathrm{J}$ Clin Biochem. 2007;22(2):90-92.

38. Polidori MC, Savino K, Alunni G, Freddio M, Senin U, Sies H, et al. Plasma lipophilic antioxidants and malondialdehyde in congestive heart failure patients: relationship to disease severity. Free Radic Biol Med. 2002;32(2):14852.

39. Yin Y, Han W, Cao Y. Association between activities of SOD, MDA and $\mathrm{Na}(+)-\mathrm{K}(+)-$ ATPase in peripheral blood of patients with acute myocardial infarction and the complication of varying degrees of arrhythmia. Hellenic $\mathbf{J}$ Cardiol. 2019;60(6):366-371.

40. Pastori D, Pignatelli P, Farcomeni A, Nocella C, Carnevale R, Violi F. Aging-related decline of glutathione peroxidase 3 and risk of cardiovascular events in patients with atrial fibrillation. 2016;5(9):e003682.

41. Negreva MN, Penev AP, Georgiev S, Aleksandrova AA. Paroxysmal atrial fibrillation: dynamics of the main antioxidant enzymes-superoxide dismutase and catalase. Folia Med (Plovdiv). 2014;56(2):96-101. 41. Dieterich S, Bieligk U, Beulich K, Hasenfuss G, Prestle J. Gene expression of antioxidative enzymes in the human heart: increased expression of catalase in the end-stage failing heart. Circulation. 2000;101(1):33-9.

42. Lenaerts I, Driesen RB, Hermida N, Holemans P, Heidbüchel H, Janssens S, et al. Role of nitric oxide and oxidative stress in a sheep model of persistent atrial fibrillation. Europace. 2013;15(5):754-60.

43.Engedal N, Žerovnik E, Rudov A, Galli F, Olivieri F, Procopio AD, et al. From Oxidative Stress Damage to Pathways, Networks, and 
Autophagy via MicroRNAs. Oxidative Medicine and Cellular Longevity. 2018;2018:1-16.

44. Wan Y, Cui R, Gu J, Zhang X, Xiang X, Liu $\mathrm{C}$, et al. Identification of Four Oxidative
Stress-Responsive MicroRNAs, miR-34a-5p, miR-1915-3p, miR-638, and miR-150-3p, in Hepatocellular Carcinoma. Oxid Med Cell Longev. 2017;2017:5189138. 